\title{
Fuzzy Universal Model Approximator for Distributed Solar Collector Field Control
}

\author{
Shahrazed Elmetennani and Taous Meriem Laleg-Kirati \\ Computer, Electrical and Mathematical Science and Engineering Division \\ King Abdullah University of Science and Technology \\ Jeddah, Saudi Arabia \\ Email: shahrazed.elmetennani@kaust.edu.sa, taousmeriem.laleg@kaust.edu.sa
}

\begin{abstract}
This paper deals with the control of concentrating parabolic solar collectors by forcing the outlet oil temperature to track a set reference. A fuzzy universal approximate model is introduced in order to accurately reproduce the behavior of the system dynamics. The proposed model is a low order state space representation derived from the partial differential equation describing the oil temperature evolution using fuzzy transform theory. The resulting set of ordinary differential equations simplifies the system analysis and the control law design and is suitable for real time control implementation. Simulation results show good performance of the proposed model.
\end{abstract}

Index Terms-Fuzzy transform, Concentrated solar collectors, Process control, Solar energy, Spatially distributed systems, Lyapunov control.

\section{INTRODUCTION}

Concentrating solar plants are utility-scale generators which convert solar energy into heat. The parabolic mirrors concentrates the sunlight to heat a fluid flowing through the receiver tube placed in the focal line of the parabola. The objective considered in parabolic trough systems is the control of the outlet oil temperature that has to evolve around a reference level. The goal is to design a control law to tune the oil flow in order to make the outlet temperature tracking a set point despite the presence of possible environmental disturbances. Mainly, the most critical disturbances are the changes in solar radiation due to weather fluctuations, and the variations in the inlet oil coming from the bottom of the storage tank. Moreover, the defined control problem is not easy to analyze because of the model complexity. Indeed, the physical distributed model describing the solar collector field is nonlinear and of infinite dimension (partial differential equation PDE) [1]-[4].

So far, many simplified models have been presented in the literature to describe the heat transfer dynamics through the collector which makes the control synthesis simpler [1] [12]. The proposed models are usually either a black box representation or a semi-discretized model approximation. The former takes advantage of experts' experience proposing a certain lumped model structure that will be used as a basis for control design [3], [7], [8]. Whereas, the latter spatially discretizes the PDE leading to a set of ordinary differential equations for finite dimensional controller design [12]. Both techniques present limitations in terms of control efficiency. Indeed, neglected dynamics lead to loss of information on the system behavior. In addition, the order of the discretized model may be very large for yielding the desired degree of approximation; resulting in complex controllers.

The exact solution of partial differential equations (PDEs) is not always easy to obtain. Therefore, for model analysis and control purposes, many numerical methods have been introduced to solve PDEs, which allow to obtain an approximation of the exact solution. Recently, fuzzy logic theory has been widely used in system modeling dealing with nonlinear systems. In most of these fuzzy designs, the fuzzy model was thought to be a universal approximator for the studied nonlinear system [13]-[21]. Particularly, the fuzzy transform theory [13] has been applied to approximate the solution of partial differential equations [14], [15].

Consequently, taking advantage of the fuzzy transform theory, a fuzzy simplified model is proposed to overcome difficulties encountered with the solar plant model. It consists of a low dimensional set of ordinary differential equations. The proposed methodology seems to be more accurate in comparison with black box models in the literature. It approximates the PDE solution using a fuzzy interpolation scheme that takes into account the infinite dimension nature of the solar collector. It is also more convenient than the semidiscretization based approaches thanks to its low order. Hence, the controller complexity is reduced and the fuzzy resulting model is suitable for real time implementation. Furthermore, the introduced fuzzy approximation has been used for output feedback controller design resorting to Lyapunov's direct method [24]. The control law has the objective to force the output of the closed loop system to track a reference by suitably adjusting the oil flow rate in the collector circuit. The outlet temperature has to follow up a specified response with a desired accuracy, where the system is subject to random irradiation and time varying inlet temperature.The proposed approximate model has been validated by comparison with the advanced PDE model simulated using semi-discretization. Then, convergence of the closed loop has been proven by simulation runs.

The paper is organized as follows. Section II describes the solar plant and presents the distributed model of the system. Section III introduces the fuzzy approximation method, followed by a model validation in Section VI. Moreover, Section V presents Lyapunov control law design. Section VI illustrates the performance of the controller with some 


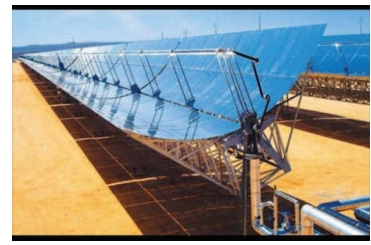

(a) An overall view of the plant [28]

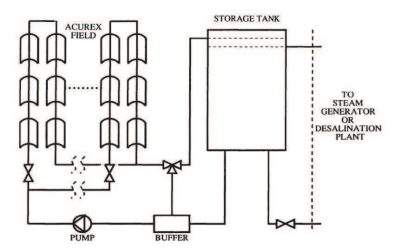

(b) A schematic diagram of the oil circuit [3]
Fig. 1. Distributed solar collector

simulations results. Finally, conclusions are given in Section VII.

\section{SOlAR COLLECTOR DESCRIPTION}

Concentrating solar collectors are industrial processes characterized by their spatially distributed behavior that depends on spatial position as well as time. A collector is formed by parabolic surface mirrors that concentrate the received sunlight in a pipe placed in the focal line of the parabola. Fig.1(a) provides an overall view of one line distributed solar collector. The oil flowing in the tube is an incompressible fluid able to support temperatures up to $300^{\circ} \mathrm{C}$ [5]. It is characterized by its very poor thermal conductivity that allows heat diffusion effects to be neglected, a fact to be exploited in the plant control design [22]. Fig. 1(b) shows a schematic diagram of the oil circuit. The oil is extracted from the bottom of a thermal storage tank, circulated through the collector field to be heated and returned to the top of the tank using a pump located at the field inlet [3]. Inside the storage tank the oil forms layers at different temperatures, which do not mix, a fact making energy storage possible [22]. From the top of this tank (hot zone inside the tank) the oil may be extracted for usage, in a desalination plant or any other thermal processes [3]. After the energy has been used, the cool oil is reinjected at the bottom of the storage tank.

A mathematical model of the solar collector has been introduced based on the energy balance. [3]-[7], [23]. However, several authors proposed a simplified version of the model, where the state representing the heat transfer is the fluid temperature [1]-[4]:

$$
\left\{\begin{array}{l}
T_{t}(x, t)+\frac{Q(t)}{S} T_{x}(x, t)=\frac{v_{0} G}{\rho c A} I(t), \\
T(0, t)=T_{i n}
\end{array}\right.
$$

for $t \in R^{+}$and $x \in[0, L]$ representing the time and the position along the collector, respectively. The variable of interest $T_{\text {out }}$ is defined as:

$$
T(L, t)=T_{\text {out }},
$$

where $T_{t}(x, t) \equiv \frac{\partial T(x, t)}{\partial t}$ and $T_{x}(x, t) \equiv \frac{\partial T(x, t)}{\partial x}$.

$T(x, t)$ designates the oil temperature at the position $x$ and the time $t . I(t)$ defines the solar irradiation evolving in time. $Q(t)$ is the oil pump volumetric flow rate that is used to control the plant. $T_{\text {in }}$ and $T_{\text {out }}$ represent the fluid temperature respectively at the positions $x=0$ and $L$. The other system parameters, according to [3], are summarized in TABLE I.

TABLE I

CONCENTRATING SOLAR COLLECTOR PARAMETERS

\begin{tabular}{llll}
\hline Symbol & Value & Measure unit & Description \\
\hline$\rho$ & 903 & $\mathrm{~kg} \mathrm{~m}^{-3}$ & Density \\
$c$ & 1820 & $\mathrm{~J} \mathrm{C}^{-1} \mathrm{~kg}^{-1}$ & Specific heat capacity \\
$S$ & 0.0006 & $\mathrm{~m}^{2}$ & Cross-sectional area \\
$v_{0}$ & $73 \%$ & & Mirror optical efficiency \\
$G$ & 1.83 & $m$ & Mirror optical aperture \\
$L$ & 172 & $m$ & Tube length \\
\hline
\end{tabular}

$f(t)=\frac{v_{0} G}{\rho c S} I(t)$ is the source term and $u(t)=\frac{Q(t)}{A}$ the system control input.

\section{NONLINEAR FUZZY APPROXIMATOR}

Taking benefits of fuzzy logic theory dealing with nonlinear and complex systems, a fuzzy approximate model is introduced in this section for the unknown temperature $T(x, t)$ along the collector tube which is described in (1). The choice of fuzzy approximation can be justified by the "Universal approximation theorem" that states [25]-[27]:

Universal approximation theorem. For any given real continuous function $g(\underline{x})$ on compact set $Q \subset \mathbb{R}^{n}$ and arbitrary $\varepsilon>0$, there exists a fuzzy system $f(\underline{x})$ such that:

$$
\sup _{\underline{x} \in Q}|f(\underline{x})-g(\underline{x})|<\varepsilon .
$$

The universe of discourse or the universal set that represents the pipe length is defined by $\Omega=[0, L]$. It is subdivided into $m$ fuzzy sets $D_{i}$ where $i=\{1, \ldots, m\}$ characterized by membership functions $\mu_{D_{i}}(x)$. These membership function take values in $\Omega$ :

$$
D_{i}=\left\{\left(x, \mu_{D_{i}}(x)\right) \mid x \in \Omega\right\} .
$$

For any $x$ as input variable to the fuzzy system, the fuzzy rules $R^{i}$ are:

$$
\begin{array}{rr}
R^{i}: \text { IF } & x \text { is } D_{i} \\
\text { THEN } & \tilde{T}(x, t)=\alpha_{i}(t) .
\end{array}
$$

$\tilde{T}(x, t)=\alpha_{i}(t)$ and $\tilde{T}\left(x_{/ \alpha}, t\right)$ are defined to be the proposed solution by the $i^{\text {th }}$ rule and the universal fuzzy approximate to describe the temperature along the space $\Omega$ at each time $t$.

The meaning of the fuzzy rule $R_{i}$ is that if the premise variables $x$ are with the fuzzy set $D_{i}$, then $\tilde{T}(x, t)$ could be represented by $\alpha_{i}(t)$. Thus the overall fuzzy approximate solution is given by:

$$
\begin{aligned}
\tilde{T}\left(x_{/ \underline{\alpha}}, t\right) & =\sum_{i=1}^{m} \alpha_{i}(t) \xi_{i}(x) \\
& =\underline{\xi}^{T}(x) \underline{\alpha}(t),
\end{aligned}
$$

where

$\underline{\alpha}(t)=\left[\alpha_{1}(t), \ldots, \alpha_{m}(t)\right]^{T}, \quad \underline{\xi}(x)=\left[\xi_{1}(x), \ldots, \xi_{m}(x)\right]^{T}$. 
The normalized membership values $\xi_{i}(x)$ are expressed as:

$$
\xi_{i}(x)=\frac{\mu_{i}(x)}{\sum_{k=1}^{m} \mu_{k}(x)}, \quad i=\{1, \ldots, m\},
$$

where the membership functions are defined as follows:

$$
\mu_{i}(x)=\exp \left[-\frac{1}{2}\left(\frac{x-a_{i}}{\sigma_{i}}\right)^{2}\right],
$$

where $a_{i}$ and $\sigma_{i}$ are the mean values and the standard deviations of the membership distributions. This particular choice was motivated by the fact that the Gaussian function is a nonlinear function in $C^{\infty}$. This makes the approximate result smooth, continuous and differentiable in $C^{\infty}$ in order to save the properties of the the exact solution defined initially by a partial differential equation.

In this work, the standard deviations are assigned as follows:

$$
\sigma_{i}=\frac{L}{m-1} .
$$

Substituting the proposed approximate solution in the physical model of (1), yields $\forall x \in \Omega$ :

$$
\begin{aligned}
& \frac{\partial}{\partial t} \tilde{T}\left(x_{/ \underline{\alpha}}, t\right)+u(t) \frac{\partial}{\partial x} \tilde{T}\left(x_{/ \underline{\alpha}}, t\right)=f(t), \\
& \underline{\xi}(x)^{T} \underline{\dot{\alpha}}(t)+u(t) \underline{\alpha}^{T}(t) \underline{\xi_{x}}(x)=f(t),
\end{aligned}
$$

such that

$$
\underline{\dot{\alpha}}(t)=\left[\dot{\alpha}_{1}(t), \ldots, \dot{\alpha}_{m}(t)\right]^{T},
$$

where the dot denotes the derivative with respect to time.

$$
\underline{\xi_{x}}(x)=\left[\xi_{x_{1}}(x), \ldots, \xi_{x_{m}}(x)\right]^{T} \text { with } \xi_{x_{i}}(x)=\frac{d}{d x} \xi_{i}(x) .
$$

For $x=0$ :

$$
\tilde{T}(0, t)=\underline{\xi}^{T}(0) \underline{\alpha}(t)=T_{i n} .
$$

Subsequently,

$$
\frac{d}{d t} \tilde{T}(0, t)=\underline{\xi}^{T}(0) \underline{\dot{\alpha}}(t)=0 .
$$

As mentioned previously, the aforementioned problem was defined on the domain $\Omega=[0, L]$. Thus, in order to perform training, we will consider a one dimensional mesh by taking $(p)$ equidistant points in the domain $[0, L]$.

Applying the design procedure (6-12) to each point of the grid yields:

$$
H \underline{\dot{\alpha}}(t)+H_{x} \underline{\alpha}(t) u(t)=F(t),
$$

with

$$
H=\left[\begin{array}{ccc}
\xi_{1}(0) & \ldots & \xi_{m}(0) \\
\xi_{1}(\Delta x) & \ldots & \xi_{m}(\Delta x) \\
\vdots & & \vdots \\
\xi_{1}(j) & \ldots & \xi_{m}(j) \\
\vdots & & \vdots \\
\xi_{1}(L) & \ldots & \xi_{m}(L)
\end{array}\right]_{p \times m}
$$

$$
\begin{gathered}
H_{x}=\left[\begin{array}{ccc}
0 & \ldots & 0 \\
\xi_{x_{1}}(\Delta x) & \ldots & \xi_{x_{m}}(\Delta x) \\
\vdots & & \vdots \\
\xi_{x_{1}}(j) & \ldots & \xi_{x_{m}}(j) \\
\vdots & & \vdots \\
\xi_{x_{1}}(L) & \ldots & \xi_{x_{m}}(L)
\end{array}\right]_{p \times m}, \\
F(t)=f(t)\left[\begin{array}{c}
0 \\
\underline{\mathbf{1}}_{(p-1) \times 1}
\end{array}\right],
\end{gathered}
$$

where $j=\{\Delta x, 2 \Delta x, \cdots,(p-1) \Delta x, L\}$ with $\Delta x=\frac{L}{p-1}$.

The resulting equation (13) can be rewritten as:

$$
\underline{\dot{\alpha}}(t)=-\left(H^{T} H\right)^{-1} H^{T} H_{x} \underline{\alpha}(t) u(t)+\left(H^{T} H\right)^{-1} H^{T} F(t) .
$$

Accordingly, the approximate dynamical model for the solar plant behavior can be expressed as a state-space representation with $\underline{\alpha}$ as the state vector of dimension $m<p$ :

$$
\left\{\begin{array}{c}
\dot{\alpha}(t)=A \underline{\alpha}(t) u(t)+B \\
y(t)=C \underline{\alpha}(t)
\end{array}\right.
$$

where $\quad A=-\left(H^{T} H\right)^{-1} H^{T} H_{x}$,

and $\quad B=\left(H^{T} H\right)^{-1} H^{T} F(t)$.

The system output representing the outlet oil temperature is

$$
y(t)=T(L, t) \text { with } C=\left[\begin{array}{lll}
\xi_{1}(L) & \ldots & \xi_{m}(L)
\end{array}\right] .
$$

Remark: The resulting model presents is characterized by its low dimension. Indeed, it is equal to $m$ the number of fuzzy sets $D_{i}$ subdividing the universal set $\Omega$. For this work, the universe of discourse $\Omega$ has been divided into 6 fuzzy sets: different trials have shown that a smaller number is unable to reproduce the distributed dynamics of the solar field accurately, while a greater number of sets will be computationally more expensive where $m=6$ is sufficient to ensure accuracy.

\section{Model VALIDATION}

An advanced model of the plant is usually used to validate the proposed approach. It consists of a finite dimensional approximation of the physical description stated by the partial differential equation in (1). Thus, simulations have run using the parameters of the ACUREX field of Plataforma Solar de Almeria (Table I). It is a solar power field situated in Tabernas in Spain, mainly used for research purposes. For more details the reader should refer to [3]. 6 fuzzy sets characterized by a Gaussian distribution have been considered along the tube length $(m=6)$. A grid of 500 points has been defined to apply the transformation technique $(p=500)$. Therefore, applying the proposed approximation method, a state space representation of dimension 6 has been derived from the partial differential equation of (1). The resulting model approximation has been compared to semi-discretized model representation of dimension 500 under variable working conditions. The results 
are shown in Fig. 2 where Fig. 3 illustrates the profiles of the inlet oil temperature and the solar irradiation and Fig. 4 the oil flow rate changes.

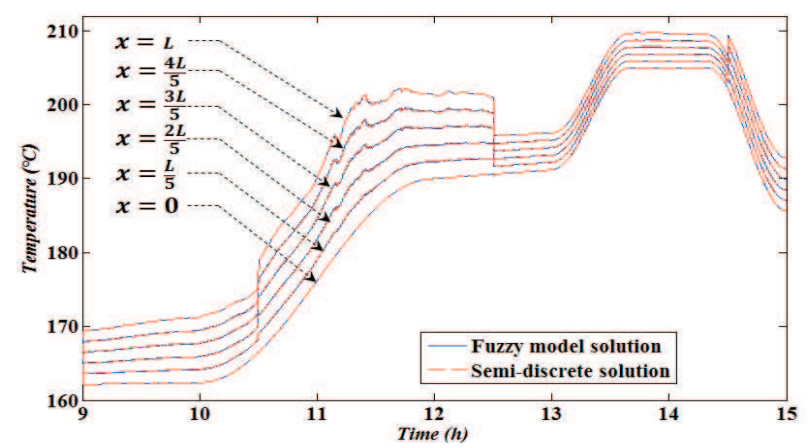

Fig. 2. Validation Results

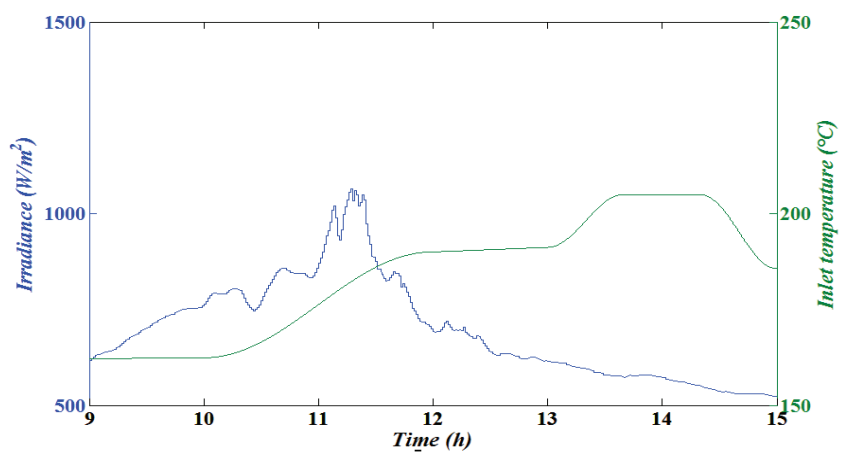

Fig. 3. Disturbances profiles

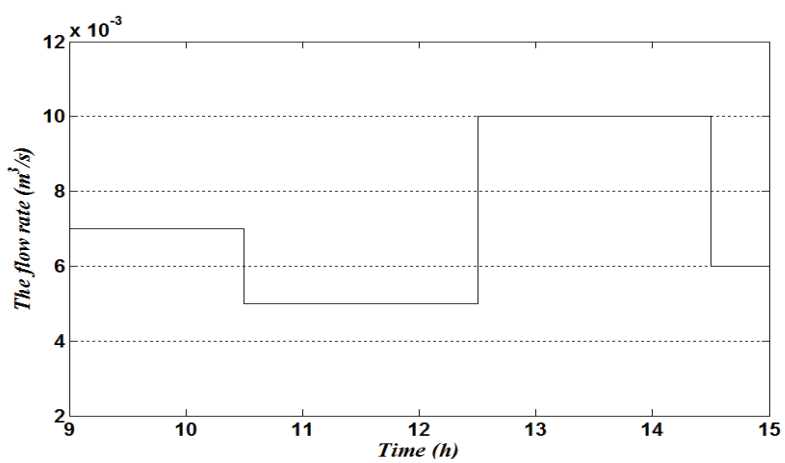

Fig. 4. Oil flow rate changes for model validation

It can be noticed that the proposed fuzzy approximate model reproduces accurately the behavior of the temperature transfer along the solar parabolic collector .

\section{LYAPUNOV NONLINEAL CONTROL}

A control system for parabolic troughs has the objective of maintaining the outlet temperature (In this case the average outlet temperature of all the parallel loops) around a set point despite the presence of external disturbances. The considered disturbances are the solar radiation variations and the irregularities in the inlet oil temperature which are supposed to be measured. The tuned variable for the solar plant control is the oil flow rate through commands to the pump. It should be pointed out that the control input is subject to physical limitations in order to respect the pump working limits and to avoid to overheat the fluid. Hence,

$$
0<u_{\text {min }}<u(t)<u_{\text {max }}, \quad \forall t>0 .
$$

Proposition 1. Assume the system dynamics obey the model (15). If the following control input is used:

$$
u(t)=\frac{(A \underline{\alpha})^{T}(K \underline{e}-B)}{(A \underline{\alpha})^{T}(A \underline{\alpha})},
$$

where $K \in \mathbb{R}^{+}$is a positive constant. Then, the closed loop system exhibits asymptotic reference tracking

$$
\lim _{t \rightarrow \infty}\|\underline{e}(t)\|=0
$$

where the tracking error is defined by:

$$
\underline{e}(t)=\underline{\alpha_{d}}-\underline{\alpha},
$$

Proof: Considering the system steady state:

$$
\frac{\partial T(x, t)}{\partial t}=0
$$

which yields:

$$
u(t) \frac{\partial T(x, t)}{\partial x}=f(x, t) .
$$

Taking the assumption that $f(x, t)$ is uniform in space (with respect to $x$ ), the temperature profile in the steady state $T_{S}(x, t)$ for a given value of $u(t)$ and $f(t)$ is written as:

$$
T_{s}(x, t)=\frac{f(t)}{u(t)} x+T_{\text {in }} \text { with } u(t) \neq 0 .
$$

Therefore, for a certain desired value of $T_{\text {out }_{d}}$, the temperature profile is expressed as:

$$
T_{s_{d}}(x)=\frac{T_{\text {out }_{d}}-T_{\text {in }}}{L} x+T_{\text {in }} .
$$

Therefore,

$$
T_{s_{d}}(x, t)=\underline{\xi}^{T}(x) \underline{\alpha_{d}}(t) .
$$

Then, the corresponding $\underline{\alpha}_{d}$ is computed by:

$$
\underline{\alpha_{d}}(t)=\left(H^{T} H\right)^{-1} H^{T} T_{S_{d}} .
$$

Once the reference vector $\alpha_{d}$ defined, the tracking error can be expressed as:

$$
\underline{e}(t)=\underline{\alpha_{d}}-\underline{\alpha},
$$

for which the dynamical evolution is managed by the equation:

$$
\underline{\dot{e}}(t)=-\underline{\dot{\alpha}}(t)=-[A \underline{\alpha}(t) u(t)+B] .
$$

The stability of the unique equilibrium point $\underline{e}=0_{p \times 1}$ of the above written error dynamics is analyzed by the following positive definite Lyapunov function:

$$
\begin{gathered}
V: \mathbb{R}^{n} \longrightarrow \mathbb{R} \\
V(t)=\frac{1}{2} \underline{e}^{T}(t) \underline{e}(t)
\end{gathered}
$$


for which the derivative function is expressed as:

$$
\begin{aligned}
\dot{V}(t) & =\underline{\dot{e}}^{T}(t) \underline{e}(t) \\
& =-\left[\begin{array}{lll}
A \underline{\alpha} & (t) u(t)+B
\end{array}\right]^{T} \underline{e}(t) .
\end{aligned}
$$

Thereafter, in order to stabilize the dynamics of the error $\underline{e}$ around its equilibrium point, the derivative function $\dot{V}$ has to be negative definite. The objective is to find the adequate control input $u(t)$ to satisfy this condition.

First, we impose that $\dot{V}$ takes the form:

$$
\dot{V}=-K \underline{e}^{T} \underline{e},
$$

which is negative definite as $K$ is a positive constant.

After that, the control input is deduced such that:

$$
\dot{V}(t)=-[A \underline{\alpha}(t) u(t)+B]^{T} \underline{e}(t)=-K \underline{e}^{T}(t) \underline{e}(t),
$$

hence:

$$
A \underline{\alpha}(t) u(t)+B=K \underline{e}(t)
$$

and finally,

$$
u(t)=\frac{(A \underline{\alpha})^{T}(K \underline{e}-B)}{(A \underline{\alpha})^{T}(A \underline{\alpha})}
$$

Applying the control law of (30) to the system of (15) results in the following derivative of Lyapunov function:

$$
\dot{V}=-K \underline{e}^{T} \underline{e},
$$

which is negative definite. This implies that starting from any initial conditions $\lim _{t \rightarrow \infty}\|\underline{e}(t)\|=0$, i.e. the reference tracking is asymptotically achieved.

\section{NUMERICAL RESULTS}

The proposed models and controllers developed for the distributed solar collectors have been performed for the ACUREX field. Therefore, the proposed simulations in this paper have also been run using the ACUREX field parameters summarized in Table I [3].

The used disturbances values have the same profiles used previously (see Fig.3). The results of the numerical simulations of the tracking error are presented in Fig. 5. The corresponding

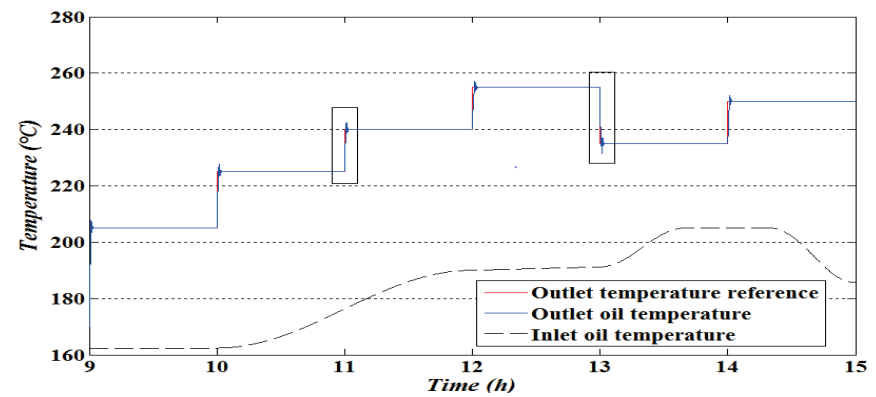

Fig. 5. Reference tracking results

generated pump command input is shown in Fig. 9.

From the presented results, it may be concluded that the Lyapunov control based on the fuzzy model ensures reference tracking despite the time varying disturbances. In addition

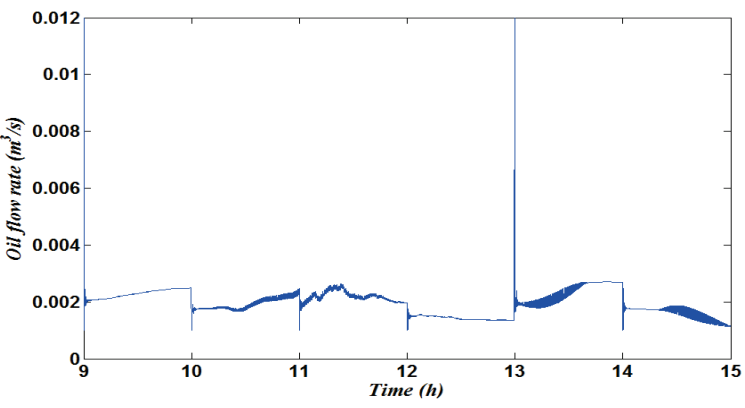

Fig. 6. Oil flow rate profile
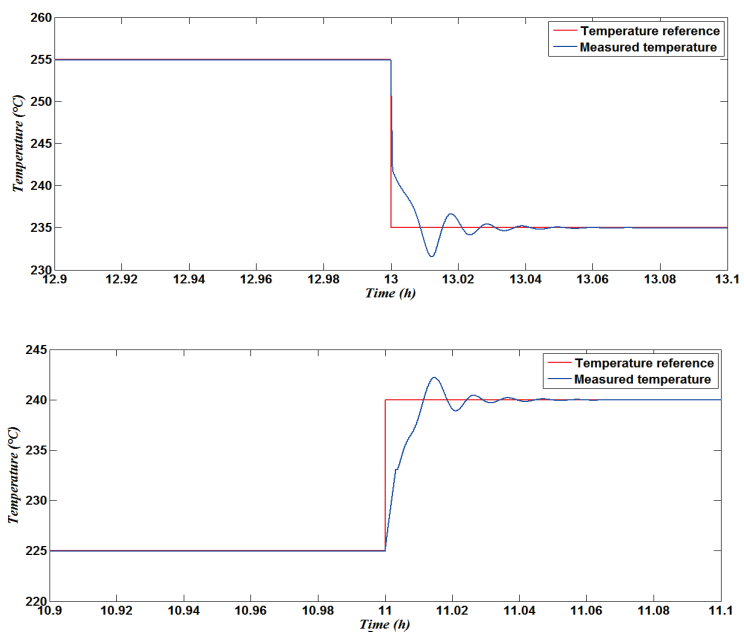

Fig. 7. Zoom sections on the system response

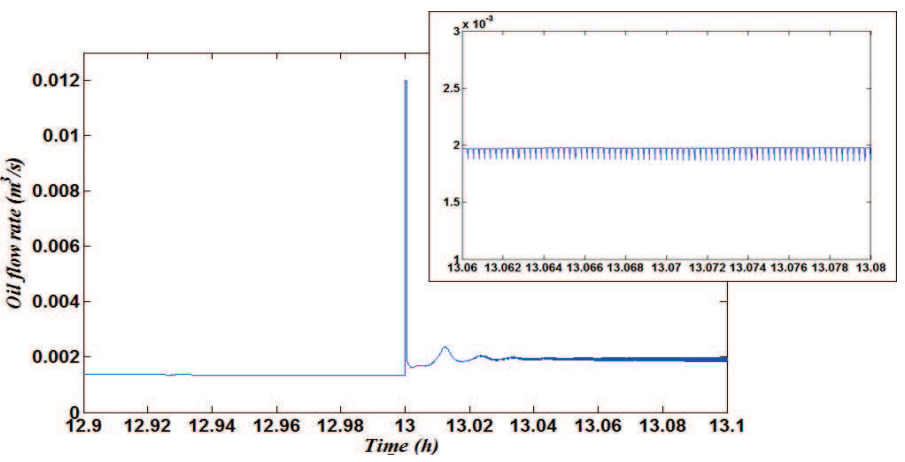

Fig. 8. Zoom on the input signal profile for decreasing temperature reference

to this, the behavior of the control law behaves regarding the changes in the temperature reference can be observed in Fig. 8 and Fig. 9. If the reference decreases suddenly, the flow rate is raised to the maximum in order to reduce the fluid temperature. The latter is then forced to follow the reference. Once around the set point value the control input evolves so that the tracking error converges to zero. Respectively, if the reference value increases, the flow rate is decreased to the minimum and then changes in order to force the outlet temperature to converge to the set point. Moreover, It can be observed that the designed control based fuzzy model allows achieving interesting performance in terms of 


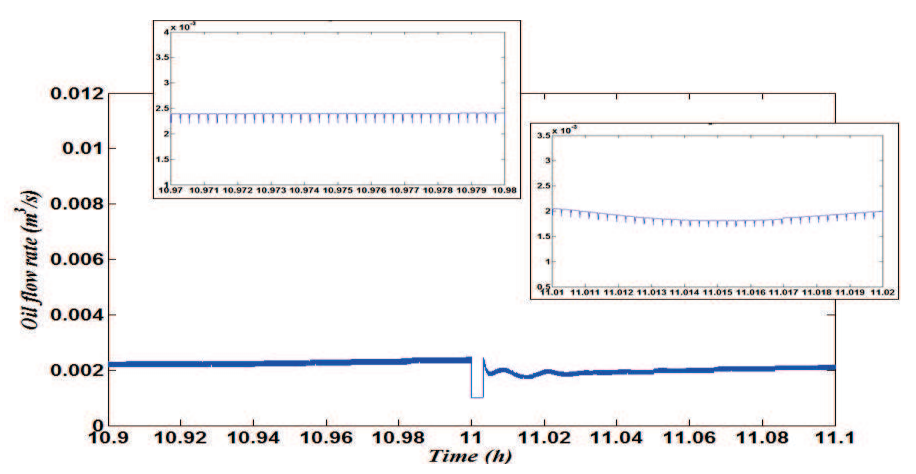

Fig. 9. Zoom on the input signal profile for increasing temperature reference

tracking precision and transient period duration. Indeed, the proposed controller forces the tracking error to converge to zero. Furthermore, the results show that the convergence time is around five minutes which represents an interesting feature for the solar plant. Fig. 10 explores the tracking error for the performed simulations.

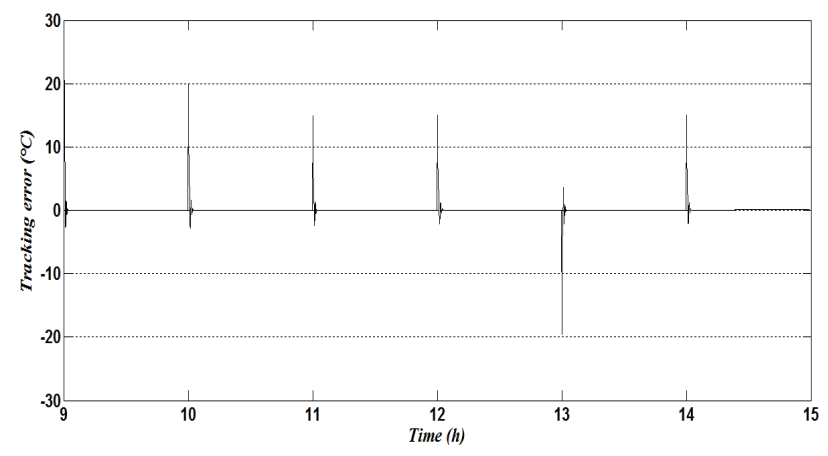

Fig. 10. Tracking error evolution

\section{CONCLUSION}

This paper presents a fuzzy universal model approximate for the distributed solar collector field. It is a state-space representation that has been derived from the partial differential equation describing the physical behavior of the solar plant using fuzzy transform theory. The proposed approximation presents many promising features since it simplifies the distributed model of the collector, by conserving the distributed character of the model. It allows to transform the PDE to a set of ordinary differential equations, which is useful for efficient control design. Another important feature is the reduction in the number of states in comparison with other methods. This makes computations simpler and easier to be implemented in real time control in terms of memory and time of computation.

\section{REFERENCES}

[1] I. Farkas, and I. Vajk, Internal model-based controller for solar plant operation, Computers and Electronics in Agriculture 49 (2005).

[2] T. A. Johansen, and C. Storaa, Energy-based control of a distributed solar collector field, Automatica 38 (2002).

[3] E. F. Camacho, M. Berenguel, and F. R. Rubio, Advances in Industrial Control, Springer, 1997.
[4] E. F. Camacho, F. R. Rubio, M. Berenguel, and L. Valenzuela, A survey on control schemes for distributed solar collector fields. Part I: Modeling and basic control approaches, Solar Energy 81 (2007).

[5] C. M. Cirre, M. Benrenguel, L. Valenzuela, E. F. Camacho, Feedback linearization control for a distributed solar collector field, Control Engineering Practice 15 (2007).

[6] M. Bernguel, E. F. Camacho, F. R. Rubio, and P. C. K. Luk, Incremental fuzzy PI control of a solar power plant, IEE Proc-Control Theory Appl, Vol 144 (6), November 1997.

[7] F. R. Rubio, M. Berenguel, and E. F. Camacho, Fuzzy Logic Control of a Solar Power Plant, IEEE transactions on Fuzzy systems, Vol 3 (4), November 1995.

[8] A. Flores, D. Saez, J. Araya, M. Berenguel, and A. Cipriano, Fuzzy predictive control of a solar power plant, IEEE transactions on fuzzy systems, Vol 13 (1) , February 2005.

[9] M. R. Arahal, M. Berenguel, E. F. Camacho, Nonlinear neural modelbased predictive control of a solar plant, Proceedings of the European Control Conference EEC'97, Brussels, Belgium, 1997.

[10] C. M. Cirre, M. Berenguel, L. Valenzuela, and R. Klempous, Reference governor optimization and control of a distributed solar collector field, European Journal of Operational Research 193 (2009).

[11] E. F. Camacho, F. R. Rubio, M. Berenguel, and L. Valenzuela, A survey on control schemes for distributed solar collector fields. Part II: Advanced control approaches, Solar Energy 81 (2007).

[12] M. Barao, J. M. Lemos, R. N. Silva, Reduced complexity adaptive nonlinear control of a distributed collector solar field, Journal of Process Control 12(2002).

[13] I. Perfilieva, Fuzzy transforms: Theory and applications, Fuzzy Sets Systems, vol. 157, pp. 993-1023, 2006.

[14] M. Stepnicka, and R.Valasek, Numerical Solution of PDE with help of Fuzzy Transform, in Proc. $14^{\text {th }}$ IEEE Int. Conf. Fuzzy Systems, pp. 1104-1109, May 2005.

[15] Y-Y Chen, Y-T Chang, and B-S Chen, Fuzzy Solutions to Partial Differential Equations: Adaptive Approach, IEEE transactions on Fuzzy Systems, vol. 17, No. 1, February 2009.

[16] H. Zhang, and D. Liu, Fuzzy Modeling and Fuzzy Control, A product of Birkhauser Basel, Springer, Series: Control Engineeering.

[17] J.-S. R. Jang, C.T. Sun, and E. Mizutani, Neuro-fuzzy and soft computing: A computational approach to learning and machine intelligence, Upper Saddle River, NJ: Prentice-Hall, 1997.

[18] B.Kosko, Fuzzy engineering, Upper Saddle River, NJ: Prentice-Hall, 1997.

[19] B. S. Chen, C. T. Tseng and H. J. Uang, Robustness design of a nonlinear dynamic systems via fuzzy linear control, IEEE transactions on Fuzzy Systems, Vol. 7 (5), October 1999.

[20] T. Leephakpreeda, Novel determination of differential-equation solutions: Universal approximation method, Journal of computational and applied mathematics, Vol. 146, 2002.

[21] J.-W. Wang, H.-N. Wu, and H.-X. Li, Distributed proportional-Spatial Derivative Control of non linear Parabolic Systems via fuzzy PDE modeling Approach, IEEE transactions on Systems, Man, and Cybernitics, Vol. 42 (3), June 2012.

[22] R. N. Silva, J. M. Lemos, and L. M. Rato, Variable sampling adaptive control of a distributed collector solar field, IEEE transactions on Control Systems Technology, Vol. 11 (5), September 2003.

[23] A.J. Gallego, F. Fele, E.F. Camacho, and L. Yebra, Obsever-based Model Predictive Control of a parabolic-trough field, Solar Energy 97(2013).

[24] H. Khalil, Nonlinear Systems, Prentice Hall PTR, 2002.

[25] M. Zhihong, X. Zhang, and L. Yanda. On universal approximation capability of fuzzy systems, Science in china, Vol. 41 (1), February 1998.

[26] B. Kosko. Fuzzy systems as universal approximators, IEEE transactions on Computers, Vol. 43 (11), November 1994.

[27] L.-X. Wang and J. M. Mendel. Fuzzy Basis Functions, Universal Approximation, and Orthogonal Least Squares Learning, IEEE transactions on Neural Networks, Vol. 3 (5), September 1992.

[28] http://www.renewablepowernews.com/ 